\title{
Flächendeckendes Managed Care: aus Erfahrung schlecht - der Blick über den Ozean
}

\author{
Marcus M. Maassen ${ }^{a}$, \\ Marco Bianchetti ${ }^{b}$ \\ a Prof. Dr. med., \\ Präsident PULSUS \\ b Dr. med. \\ Augentagesklinik Sursee
}

Aktuell sind leider die Auswirkungen eines flächendeckenden Managed-Care-Systems auf die Praxis wenig bekannt. Da ein flächendeckendes ManagedCare-System in der Schweiz als Anschauungsbeispiel nur eingeschränkt zur Verfügung steht, sind somit die praktischen Erfahrungen in der Schweiz begrenzt und haben mit der Realität eines flächendeckend netzwerkorganisierten Gesundheitssystems nichts zu tun. Auf der Suche nach einem praktischen Anschauungsbeispiel sei daher der Blick über den Ozean als Horizonterweiterung erlaubt. der Spezialärzte höhere Gewinne erzielen. Höhere Gewinne werden erzielt, aber weder bei Grundversorgern noch bei Spezialisten, sondern nur bei den Managed-Care-Versicherern. Leidtragende sind aber nicht nur die gesamte Ärzteschaft, die sich nun auf 3-Minuten-Konsultationen einstellen musste, sondern auch die Patienten. Aus erster Hand berichtet dazu ein amerikanischer Professor von der Universität von Newark, New Jersey, über zwei Fälle:

Prof. Zarbin: «Ein Patient mit einem Netzhautloch kam zu einem meiner Kollegen. Dieser diagnos-

\section{Dass sich der Kampf für die freie Arztwahl lohnt, wurde eindrücklich mit dem Referendum gegen die Managed-Care-Vorlage unter Beweis gestellt.}

In den letzten Jahrzehnten ist flächendeckend in den USA das Managed-Care-Netzwerk im Gesundheitssystem eingeführt worden. Kernpunkte in diesem flächendeckenden Managed-Care-System sind, dass der Versicherte eine Krankenversicherung auswählt und eine reduzierte Prämie zahlt, wenn die Krankenversicherung den Patienten über eine Managed-Care-Organisation behandeln lässt. Ziel der Krankenversicherung ist dabei, eine Capitation einzuhalten, in der pro Versichertem die Ausgaben möglichst niedrig budgetiert werden. Dies wird durch die Einschränkung der freien Arztwahl erreicht. Die Erstanlaufstelle Spezialarzt wird in der Grundversicherung verboten. Durch sogenannte Leitlinien werden konkrete Handlungsempfehlungen gegeben. Der Zugang zu medizinischen Leistungen wird durch administrative Hindernisse erschwert. In der Konsequenz haben die Krankenkassen einen «Super-Macht-Status» erhalten. Es entbehrt nicht einer gewissen Pikanterie, dass der hoffnungsvolle Anspruch einer qualitätsverbessernden Medizin erreicht werden soll, indem Anreize gestellt werden, nichts zu verordnen und nicht zu überweisen.

Die Einführung des Systems wurde mit denselben taktischen Manövern bewerkstelligt, die nun auch in der Schweiz zum Einsatz kommen. Es wurden Einkommensunterschiede zwischen Grundversorgern und Spezialisten zum Anlass genommen, den Grundversorgern Hoffnung zu machen, sie würden in Managed Care durch Einkommensverluste tizierte das behandlungsbedürftige Loch und telefonierte sofort der Versicherung des Patienten, ob sie die Laserbehandlung übernehme. Nach einiger Wartezeit gelangte er an die zuständige Person und bekam einen Berechtigungs-Code für die Laserbehandlung. Einige Wochen später aber verweigerte die Versicherung die Bezahlung der Behandlungskosten trotz Berechtigungs-Code. Der Arzt hatte die falsche Telefonnummer angerufen. Korrekterweise hätte er eine Nummer für Notfallbehandlungen anrufen sollen. Ein akademischer Kollege aus der Schweiz musste bei seinem Sohn eine Operation durchführen lassen. Er war in den USA in einer Managed-Care-Versicherung, die den ausgewiesenen Spezialisten für diese Operation nicht unter Vertrag hatte. Der Kollege ist dann mit seinem Sohn zurück in die Schweiz geflogen, um die Operation dort durchführen zu lassen. Ich habe mir dabei gedacht, die Schweiz habe wirklich ein ausgezeichnetes Gesundheitssystem, mit freiem Zugang zu allen Ärzten.»

Professor Zarbin meinte: «Kämpft für die freie Arztwahl, denn unter den Vorgaben der Krankenversicherer wird die Qualität der medizinischen Leistungen schlechter trotz Qualitäts-Indices, die das Gegenteil zu beweisen versuchen. Ärzte und Patienten sind in diesem System gemeinsame Verlierer.»

Dass sich der Kampf für die freie Arztwahl lohnt, wurde eindrücklich mit dem Referendum gegen die Managed-Care-Vorlage unter Beweis gestellt. Mit 
Schreiben vom 15. Februar 2012 hat die Bundeskanzlei bestätigt: «Das Referendum gegen die Änderung vom 30. September 2011 des Bundesgesetzes über die Krankenversicherung (KVG) (Managed Care) ist zustande gekommen, da es die nach Artikel $141 \mathrm{Ab}$ satz 1 der Bundesverfassung verlangten 50000 gültigen Stimmen aufweist. Von insgesamt 133185 eingereichten Unterschriften sind 131158 gültig.»

Tabelle 1

Referendum gegen die Änderung vom 30. September 2011 des Bundesgesetzes über die Krankenversicherung (KVG) (Managed Care). Unterschriften nach Kantonen.

\begin{tabular}{|c|c|c|}
\hline Kantone & gültige Unterschriften & ungültige Unterschriften \\
\hline Zürich & 17949 & 127 \\
\hline Bern & 12302 & 146 \\
\hline Luzern & 10284 & 223 \\
\hline Uri & 574 & 13 \\
\hline Schwyz & 2385 & 40 \\
\hline Obwalden & 369 & 2 \\
\hline Nidwalden & 653 & 1 \\
\hline Glarus & 239 & 3 \\
\hline Zug & 1768 & 18 \\
\hline Freiburg & 3275 & 18 \\
\hline Solothurn & 2342 & 63 \\
\hline Basel-Stadt & 6265 & 59 \\
\hline Basel-Landschaft & 7945 & 202 \\
\hline Schaffhausen & 828 & 4 \\
\hline Appenzell A.-Rh. & 381 & 6 \\
\hline Appenzell I.-Rh. & 140 & 0 \\
\hline St. Gallen & 4731 & 30 \\
\hline Graubünden & 1576 & 16 \\
\hline Aargau & 5130 & 68 \\
\hline Thurgau & 1268 & 26 \\
\hline Tessin & 6057 & 55 \\
\hline Waadt & 23392 & 754 \\
\hline Wallis & 4672 & 34 \\
\hline Neuenburg & 2895 & 42 \\
\hline Genf & 12867 & 72 \\
\hline Jura & 871 & 5 \\
\hline Schweiz & 131158 & 2027 \\
\hline
\end{tabular}

Schliesslich ist das tatkräftig vorangetriebene Engagement und die erhaltene Unterstützung für das Referendum ein Vertrauensbeweis auch in die Arbeit des Vereins PULSUS, bei dem 30\% der Mitglieder Hausärzte sind. Wir möchten uns, geschätzte Kolleginnen und Kollegen, bei Ihnen ganz herzlich bedanken. Wir danken Ihnen - der FMH, der fmCh, der FMP, der PSICA, dem Verein für freie Arztwahl und dem VPOD - VSAO, aber insbesondere auch den kantonalen Gesellschaften Baselland, Basel-Stadt, Schaffhausen, Genf und Schwyz sowie den Fachgesellschaften Dermatologie, Ophthalmologie und Otorhinolaryngologie - Hals- und Gesichtschirurgie und den Hunderten Hausarztpraxen in der gesamten Schweiz - für die Unterstützung. Im Ergebnis wurde durch Ihr tatkräftiges Engagement das in Tabelle 1 dargestellte, ausgezeichnete Referendumsresultat erzielt.

«Objektiv ist das Referendum zu befürworten», schreibt Dr. Andreas Keusch aus Pfäffikon (medvice[at]bluewin.ch) in einer aktuellen, ausgezeichneten Übersichtsarbeit zur Managed-CareVorlage (Förderung von integrierten Versorgungsnetzen zur Verbesserung der Qualität und Effizienz im Schweizerischen Gesundheitswesen: - «Wirtschaftliche» Mogelpackung oder «Zweckmässige» Qualitätsförderung?!). Herr Dr. Keusch argumentiert überzeugend, indem er formuliert: «Um wieder auf die eingangs erwähnte Aussage «Integrierte Ärzte-Netzwerke bieten beste Qualität zu tieferen Kosten» von Frau Nationalrätin Prisca Birrer-Heimo und den MC-Befürwortern zurückzukommen, fehlt es somit an einer substantiellen Grundlage zum Beleg der vorliegenden medizinischen Qualität des Leistungsangebotes in integrierten Netzwerken.» Die Studie steht als Download auf www.pulsus.info zur Verfügung.

Liebe Kolleginnen und Kollegen, auch wir sind überzeugt, dass ein Managed-Care-Zwangssystem, das die Abschaffung der freien Arztwahl in der Grundversicherung zur Folge hat, die Zerrüttung der Ärzteschaft untereinander fördert und alle Macht den Krankenkassen zuteilt, nicht die Basis für ein zukünftiges Schweizer Gesundheitssystem sein kann. 